\title{
COMMUTATIVE RINGS WHOSE QUOTIENTS ARE GOLDIE
}

\author{
by VICTOR P. CAMILLO
}

(Received 11 February, 1974; revised 12 September, 1974)

All rings considered here have units. A (non-commutative) ring is right Goldie if it has no infinite direct sums of right ideals and has the ascending chain condition on annihilator right ideals. A right ideal $A$ is an annihilator if it is of the form $A=\{a \in R \mid x a=0$ for all $x \in X\}$, where $X$ is some subset of $R$. Naturally, any noetherian ring is Goldie, but so is any commutative domain, so that the converse is not true. On the other hand, since any quotient ring of a noetherian ring is noetherian, it is true that every quotient is Goldie. A reasonable question therefore is the following: must a ring, such that every quotient ring is Goldie, be noetherian? We prove the following theorem:

THEOREM. A commutative ring is noetherian if and only if every quotient is Goldie.

The hypothesis that every quotient be Goldie is very strong and would seem likely to yield an elementary proof of the theorem. We have not been able to do this, however, without the use of a strong theorem of Shock.

A module $M$ is a max module if every submodule has a maximal submodule. Shock has proved:

THEOREM (Shock). A non-finitely generated max module has a quotient with infinite socle and zero Jacobson radical [5, Theorem 2.8].

We also need some ideas on $T$-nilpotence. A right ideal $A$ in a ring $R$ is left $T$-nilpotent if, given an infinite sequence $\left\{m_{1}, m_{2}, \ldots, m_{n}, \ldots\right\}$ of elements of $A$, there is an integer $N$ with $m_{M} \ldots m_{1}=0$. It is well known that if $A$ is such a right ideal and $N \neq 0$ is a right $R$ module, then $N A \neq N$.

If in the above paragraph, $A$ were a two-sided ideal having the property that every $R / A$ module had a maximal submodule, then since $N / N A$ is non-zero, it would be the case that every $R$ module would have a maximal submodule. In particular, this would be the case if $R$ were commutative and $A$ were a maximal ideal.

To prove the theorem, we first state a lemma :

LEMMA. If $R$ is a commutative ring with unit, having essential, simple socle, and having the ascending chain condition on annihilators of single elements, then $R$ has a $T$-nilpotent maximal ideal.

Proof. Let $S=s R$ be the socle of $R$, and let $M=$ ann $s$. Let $\left\{m_{1}, m_{2}, \ldots, m_{n} \ldots\right\}$ be a collection of elements in $M$. By the a.c.c. on annihilators, there is an integer $N$ with $\operatorname{ann}\left(m_{1} \ldots m_{N}\right)=\operatorname{ann}\left(m_{1} \ldots m_{N+1}\right)$. This says that ann $m_{N+1} \cap\left(m_{1} \ldots m_{N}\right) R=0$. But, since $S$ is essential, any two ideals in $R$ have non-zero intersection. Further, $m_{N+1} S=0$, so that ann $m_{N+1} \neq 0$. Thus $\left(m_{1} \ldots m_{N}\right) R=0$, so that $m_{1} \ldots m_{N}=0$. 
Proof of the theorem. We show that if $R$ is a commutative fully Goldie ring, then $R_{R}$ is a max module. Let $A$ be any ideal in $R$, and let $x \in A, x \neq 0$. Let $B$ be an ideal, maximal with respect to excluding $x$. Then, as is easily shown, the ring $R / B$ has simple essential socle generated by $x+B$. Further, $A+B / B$ is a non-zero module over $R / B$. By the lemma, $R / B$ has a $T$-nilpotent maximal ideal and, by the observations preceding the theorem, every module over $R / B$ has a maximal submodule, so that $A+B / B$ does. This corresponds to a maximal submodule $M$ of $A+B$ containing $B$. Then $M \ngtr A$, so that $M \cap A$ is a maximal submodule of $A$.

Now, if $A$ is a non-finitely generated ideal in $R, A$ is a max module, so that, by Shock's Theorem, $A$ would have a quotient with infinite socle, contradicting the Goldie hypothesis.

Remark. Generalizations to the non-commutative case seem difficult. If one restricts oneself only to quotient rings, there may not be enough two-sided ideals for the hypothesis to have much strength. If one considers cyclic modules, it is not clear what an annihilator submodule is. Perhaps it could be one which is the intersection of the kernels of a collection of homomorphisms?

Rings having the property that $R / I$ is Goldie for all two-sided ideals are studied in [3], $[4]$, and [6].

\section{REFERENCES}

1. F. Anderson and K. Fuller, Rings and Modules of Categories, Springer (to appear).

2. A. W. Goldie, Rings with Maximum Condition, Lecture notes (Yale University Press, 1961).

3. A. V. Jategonkar, Left Principal Ideal Rings, Lecture Notes in Mathematics, Springer, 1970.

4. J. C. Robson, Artinian Quotient Rings, Proc. London Math. Soc. (3) 17 (1967), 600-616.

5. R. Shock, Dual Generalizations of the Artinian and Noetherian Conditions, Pacific J. Math. (to appear).

6. L. W. Small, Orders in Artinian Rings, J. Algebra, 4 (1966), 13-41.

\section{UNIVERSITY OF IOWA}

IOWA CITY

IOWA 52240, U.S.A. 